\title{
Equivariant indices of vector fields and 1-forms
}

\author{
Wolfgang Ebeling • Sabir M. Gusein-Zade
}

Received: 7 October 2014 / Accepted: 13 February 2015 / Published online: 5 March 2015

(C) Springer International Publishing AG 2015

\begin{abstract}
Equivariant versions of the radial index and of the GSV-index of a vector field or a 1-form on a singular variety with an action of a finite group are defined. They have values in the Burnside ring of the group. Poincaré-Hopf type theorems for them are proven and some of their properties are described.
\end{abstract}

Keywords Singularities · Vector fields · 1-Forms · Finite group action · Index · Burnside ring

Mathematics Subject Classification $\quad 14 \mathrm{~B} 05 \cdot 58 \mathrm{~K} 45 \cdot 58 \mathrm{~A} 10 \cdot 58 \mathrm{E} 40 \cdot 19 \mathrm{~A} 22$

\section{Introduction}

The classical notion of the index of an isolated singular point (zero) of a vector field has several generalizations to vector fields and 1-forms on singular varieties. In particular, there were defined the GSV-index of a vector field on an isolated hypersurface [16] or complete intersection singularity [24], the Euler obstruction of a 1-form [22], the

Partially supported by the DFG (Mercator fellowship, Eb 102/8-1), the Russian government grant 11.G34.31.0005, RFBR-13-01-00755, and NSh-4850.2012.1.

W. Ebeling $(\bowtie)$

Institut für Algebraische Geometrie, Leibniz Universität Hannover,

Postfach 6009, 30060 Hannover, Germany

e-mail: ebeling@math.uni-hannover.de

S. M. Gusein-Zade

Faculty of Mechanics and Mathematics, Moscow State University,

GSP-1, Moscow 119991, Russia

e-mail: sabir@mccme.ru 
homological index of a vector field [15] or of a 1-form [14], the radial index of a vector field or of a 1-form, etc.

The radial index of an isolated singular point of a (stratified) vector field or of a 1 -form on a singular variety was defined in $[9,11]$, following earlier ideas of $[8,23]$. Initially it was defined for vector fields and 1-forms on analytic varieties. However without any difference one can consider the radial index on closed (real) subanalytic sets (as noticed in [7]). The sum of the radial indices of a vector field or of a 1-form with isolated singular points on a compact variety is equal to the Euler characteristic of the variety. Therefore the (radial) index can be considered as a localization of the Euler characteristic (on a singular variety). Surveys on indices of vector fields and 1 -forms on singular varieties can be found in $[2,11]$.

There are several equivariant versions of the Euler characteristic for spaces with actions of a finite group $G$. First, one can consider the Euler characteristic of the quotient space. One can define an equivariant Euler characteristic as an element of the representation ring of the group $G$; see, e.g., $[4,26]$. There exists the so-called orbifold Euler characteristic (with values in the ring $\mathbb{Z}$ of integers) offered by physicists $[5,6,20]$ and its higher order generalizations [1,3]. However a more general concept is the equivariant Euler characteristic defined as an element of the Burnside ring $B(G)$ of the group $G[4,25]$. The other versions of the Euler characteristic mentioned above are specializations of this one.

This makes it reasonable to try to define indices of singular points of vector fields and of 1 -forms on $G$-varieties as elements of the Burnside ring $B(G)$ so that they will be localizations of the equivariant Euler characteristic. Their specializations will give other equivariant versions of the indices, say, as an element of the ring of representations or an orbifold version (with values in $\mathbb{Z}$ ). For vector fields on (smooth) $G$-manifolds this was done in [21].

In this paper we define equivariant versions with values in the Burnside ring of the radial index and of the GSV-index, prove Poincaré-Hopf type theorems for them and describe some of their properties.

In Sect. 2 we recall the concepts of the radial index and of the GSV-index in the usual (non-equivariant) setting. In Sect. 3 we give definitions of the equivariant Euler characteristic, of the orbifold Euler characteristic and of its higher order generalizations. In Sect. 4 we discuss an equivariant version of the radial index. Sect. 5 is devoted to an equivariant version of the GSV-index.

\section{Indices of vector fields and 1-forms}

Let $(V, 0)$ be a germ of a closed (real) subanalytic set. We assume $(V, 0)$ to be embedded into $\left(\mathbb{R}^{N}, 0\right)$. Let $V=\bigcup_{i=1}^{q} V_{i}$ be a subanalytic Whitney stratification of $V$. A (continuous) stratified vector field on $V=\bigcup_{i=1}^{q} V_{i}$ is a vector field such that, at each point $p$ of $V$, it is tangent to the stratum containing $p$ [2].

Let $B_{\varepsilon}$ be a closed ball (in the ambient space $\mathbb{R}^{N}$ ) around the origin (small enough so that the sphere $S_{\varepsilon}=\partial B_{\varepsilon}$ intersects all the strata $V_{i}$ transversally). Let $L=V \cap S_{\varepsilon}$ be the link of $V$. There exists a diffeomorphism $h$ of $V \cap B_{\varepsilon}$ with the cone $C L=$ $L \times[0,1] / L \times\{0\}$ over $L$ being the identity on $L=L \times\{1\}$. (A diffeomorphism 
of stratified varieties is a homeomorphism which respects the stratification and is a diffeomorphism on each stratum.) A vector field $Y$ on $(V, 0)$ is called radial if, for a certain diffeomorphism $h$, it is identified with the vector field $\partial / \partial t$ on $C L$.

Let $p \in V$, let $V_{(p)}=V_{i}$ be the stratum containing $p, \operatorname{dim} V_{i}=k$, and let $Y$ be a stratified vector field on $V$ in a neighbourhood of the point $p$. Let $N_{i}$ be a normal slice of $V$ to the stratum $V_{i}$ at the point $p$. There exists a diffeomorphism $h$ from a neighbourhood of $p$ in $V$ to the product $U_{i}(p) \times N_{i}$, where $U_{i}(p)$ is a neighbourhood of $p$ in $V_{i}$, which is the identity on $U_{i}(p)$. The vector field $Y$ is called a radial extension of the vector field $Y_{\mid V_{i}}$ if there exists a diffeomorphism $h$ which identifies $Y$ with the vector field on $U_{i}(p) \times N_{i}$ of the form $\left(Y_{\mid V_{i}}, 0\right)+\left(0, Y_{N_{i}}^{\mathrm{rad}}\right)$, where $Y_{N_{i}}^{\mathrm{rad}}$ is a radial vector field on $N_{i}$ [2].

Let $X$ be a stratified vector field on $(V, 0)$ with an isolated singular point (zero) at the origin. One can show that there exists a (continuous) stratified vector field $\widetilde{X}$ on $V$ such that:

(a) the vector field $\widetilde{X}$ coincides with $X$ on a neighbourhood of the intersection of $V$ with the sphere $S_{\varepsilon}=\partial B_{\varepsilon}$ of a small radius $\varepsilon$ around the origin;

(b) the vector field has a finite number of singular points (zeros);

(c) in a neighbourhood of each singular point $p \in V \cap B_{\varepsilon}, p \in V_{i}$, the vector field $\widetilde{X}$ is a radial extension of its restriction to the stratum $V_{i}$.

The radial index $\operatorname{ind}_{\mathrm{rad}}(X ; V, 0)$ of the vector field $X$ on $V$ at the origin is

$$
\sum_{p \in \operatorname{Sing} \tilde{X}} \operatorname{ind}\left(\widetilde{X}_{\mid V_{(p)}} ; V_{(p)}, p\right)
$$

where ind $\left(\widetilde{X}_{\mid V_{(p)}}, V_{(p)}, p\right)$ is the usual index of the restriction of the vector field $\widetilde{X}$ to the smooth manifold $V_{(p)}$.

Now let $\omega$ be a (continuous) 1 -form on $(V, 0)$ (i.e. the restriction to $V$ of a 1 -form defined in a neighbourhood of 0 in $\mathbb{R}^{N}$ ). Let $V=\bigcup_{i=1}^{q} V_{i}$ be a subanalytic Whitney stratification of $V$. A point $p \in V$ is a singular point of $\omega$ if the restriction of $\omega$ to the stratum $V_{(p)}$ containing $p$ vanishes at the point $p$. A 1 -form $\eta$ on $(V, 0)$ is called radial if its restriction to any (parametrized) analytic curve $\varphi:\left(\mathbb{R}_{\geq 0}, 0\right) \rightarrow(V, 0)$ is positive on the vector $\varphi_{*} \partial / \partial t$ in a punctured neighbourhood of zero. As an example, one can take $d\|\cdot\|^{2}$, where $\|\cdot\|$ is the Euclidean norm in $\mathbb{R}^{N}$.

For a point $p, p \in V_{i}=V_{(p)}, \operatorname{dim} V_{(p)}=k$, there exists a (local) analytic diffeomorphism $h:\left(\mathbb{R}^{N}, \mathbb{R}^{k}, 0\right) \rightarrow\left(\mathbb{R}^{N}, V_{(p)}, p\right)$. A 1 -form $\eta$ is called a radial extension of the 1-form $\eta_{\mid V_{(p)}}$ if there exists a diffeomorphism $h$ which identifies $\eta$ with the restriction to $V$ of the 1 -form $\pi_{1}^{*} \eta_{\mid V_{(p)}}+\pi_{2}^{*} \eta_{\mathbb{R}^{N-k}, 0}^{\text {rad }}$, where $\pi_{1}$ and $\pi_{2}$ are the projections of $\mathbb{R}^{N}$ to $\mathbb{R}^{k}$ and $\mathbb{R}^{N-k}$ respectively, $\eta_{\mathbb{R}^{N-k}, 0}^{\text {rad }}$ is a radial 1 -form on $\left(\mathbb{R}^{N-k}, 0\right)$.

For a 1 -form $\omega$ on $(V, 0)$ with an isolated singular point at the origin there exists a 1 -form $\widetilde{\omega}$ on $V$ which possesses the obvious analogues of the properties (a)-(c) of the vector field $\widetilde{X}$ above. The radial index of $\omega$ is

$$
\operatorname{ind}_{\operatorname{rad}}(\omega ; V, 0)=\sum_{p \in \operatorname{Sing} \widetilde{\omega}} \operatorname{ind}\left(\widetilde{\omega}_{\mid V_{(p)}} ; V_{(p)}, p\right)
$$


There is a one-to-one correspondence between complex valued and real valued 1 -forms on a complex analytic variety. The real 1-form $\alpha$ corresponding to a complex 1 -form $\omega$ is just its real part: $\alpha=\operatorname{Re} \omega$. The 1 -form $\omega$ can be restored from $\alpha$ by the equation $\omega(u)=\alpha(u)-\alpha(i u) i$ ( $u$ is a tangent vector to the ambient space). Therefore, for a complex 1-form on a complex analytic variety, its radial index is defined as the radial index of its real part. For a complex 1 -form on a $d$-dimensional complex analytic manifold, this index differs from the usual one by the sign $(-1)^{d}$.

The radial index of a vector field or of a 1-form is defined as the sum of the indices of an appropriate deformation of it on the strata (smooth manifolds). The sum is the same for a stratification and for a refinement of it. This implies that the radial index (and also its equivariant version below) does not depend on the stratification and thus is well-defined. (For a vector field one can consider the intersection of two stratifications. For a 1-form one can consider the minimal Whitney stratification of the variety.)

Let $(V, 0)$ be a germ of a subanalytic set, let $V=\bigcup_{i=1}^{q} V_{i}$ be a (subanalytic) Whitney stratification of $V$, and let $X$ or $\omega$ be a stratified vector field or a 1-form on $(V, 0)$ respectively with an isolated singular point at the origin. One can show that the radial index $\operatorname{ind}_{\mathrm{rad}}(X ; V, 0)$ or $\operatorname{ind}_{\mathrm{rad}}(\omega ; V, 0)$ can be distributed between the strata $V_{i}$ in the following sense. The index is the number of singular points of an appropriate deformation $\widetilde{X}$ or $\widetilde{\omega}$ of $X$ or $\omega$ respectively counted with multiplicities.

Proposition 2.1 The number of singular points (counted with multiplicities) of the vector field $\widetilde{X}$ or of a 1-form $\widetilde{\omega}$ on a fixed stratum $V_{i}$ does not depend on the choice of the vector field $\widetilde{X}$ or of the 1-form $\widetilde{\omega}$ respectively (and therefore only depends on $X$ or $\omega$ respectively).

Proof We shall write the proof in terms of vector fields. The proof for 1 -forms is the same. We shall follow this scheme in the sequel as well.

Let $\widetilde{X}_{1}$ and $\widetilde{X}_{2}$ be two deformations of $X$ with the necessary properties. Let $W_{i}$ be the complement in $V_{i}$ of a small tubular neighbourhood of its boundary $\partial V_{i}$. The intersection $W_{i} \cap B_{\varepsilon}$ (after an appropriate smoothing at $\partial W_{i} \cap S_{\varepsilon}$ ) is a manifold with boundary. The vector fields $\widetilde{X}_{1}$ and $\widetilde{X}_{2}$ coincide on the part $W_{i} \cap S_{\varepsilon}$ of the boundary of $W_{i}$. On the remaining part of the boundary these vector fields are homotopic, in the class of non-vanishing vector fields, to vector fields pointing inside $W_{i}$ and therefore are homotopic to each other (in the same class of vector fields non-vanishing on the boundary). This implies that the numbers of the singular points of the vector fields $\widetilde{X}_{1}$ and $\widetilde{X}_{2}$ on $W_{i}$ counted with multiplicities coincide.

Another argument for the statement can be the following one. The closure $\overline{V_{i}}$ of the stratum $V_{i}$ and its boundary $\overline{V_{i}} \backslash V_{i}$ are closed subanalytic varieties. The numbers of the singular points of the vector field $\widetilde{X}$ on $\overline{V_{i}}$ and on $\overline{V_{i}} \backslash V_{i}$ (counted with multiplicities) are equal to the indices $\operatorname{ind}_{\mathrm{rad}}\left(X ; \overline{V_{i}}, 0\right)$ and $\operatorname{ind}_{\mathrm{rad}}\left(X ; \overline{V_{i}} \backslash V_{i}, 0\right)$ respectively (and therefore are well-defined). The number of the singular points of $\widetilde{X}$ on $V_{i}$ is the difference

$$
\operatorname{ind}_{\mathrm{rad}}\left(X ; \overline{V_{i}}, 0\right)-\operatorname{ind}_{\mathrm{rad}}\left(X ; \overline{V_{i}} \backslash V_{i}, 0\right) .
$$


This means that the radial index can be defined for each stratum $V_{i}$ so that

$$
\operatorname{ind}_{\operatorname{rad}}(X ; V, 0)=\sum_{i=1}^{q} \operatorname{ind}_{\mathrm{rad}}\left(X ; V_{i}, 0\right) .
$$

Moreover the radial index can be defined as a linear map from the group of constructible, integer valued functions on $V$ to $\mathbb{Z}$. (The radial index $\operatorname{ind}_{\text {rad }}(X ; V, 0)$ is the image of the constant function 1.)

There is also the notion of the GSV-index of a vector field or a 1-form on a complex analytic complete intersection singularity. We shall discuss this notion (and therefore its $G$-equivariant version below) in the generality considered in [2]. Let $f_{1}, \ldots, f_{k}$ be holomorphic function germs on $\left(\mathbb{C}^{n}, 0\right)$ defining a complete intersection singularity $(V, 0)=\left\{f_{1}=\cdots=f_{k}=0\right\}$. We assume that a neighbourhood of the origin in $\mathbb{C}^{n}$ permits a Whitney stratification adapted to $V$ and satisfying the Thom $a_{f}$ condition. This holds, in particular, if $(V, 0)$ is an isolated complete intersection singularity (ICIS) or if it is a hypersurface, i.e. $k=1$. Let $F$ be the map $F=\left(f_{1}, \ldots, f_{k}\right):\left(\mathbb{C}^{n}, 0\right) \rightarrow\left(\mathbb{C}^{k}, 0\right)$ and let $\Delta \subset\left(\mathbb{C}^{k}, 0\right)$ be the discriminant of $F$. For $0<\delta \ll \varepsilon$ small enough the restriction of the map $F$ to $B_{\varepsilon}^{2 n}$ is a locally trivial fibration over $B_{\delta}^{2 k} \backslash \Delta\left(B_{\varepsilon}^{2 n}\right.$ and $B_{\delta}^{2 k}$ are the closed balls of radii $\varepsilon$ and $\delta$ around the origin in $\mathbb{C}^{n}$ and in $\mathbb{C}^{k}$ respectively). For $\underline{\delta}=\left(\delta_{1}, \ldots, \delta_{k}\right) \in B_{\delta}^{2 k} \backslash \Delta$, the fibre $M_{\underline{\delta}}=F^{-1}(\underline{\delta}) \cap B_{\varepsilon}^{2 n}$ of this fibration is a non-singular complex manifold with the boundary $\partial M_{\underline{\delta}}=F^{-1}(\underline{\delta}) \cap S_{\varepsilon}^{2 n-1}$ - the Milnor fibre of $F$ or of $(V, 0)$ (the set $S_{\varepsilon}^{2 n-1}=\partial B_{\varepsilon}^{2 n}$ is the sphere of radius $\varepsilon$ around the origin in $\left.\mathbb{C}^{n}\right)$.

Let $X$ be a stratified vector field on $(V, 0)$ with an isolated singular point at the origin. In particular, the restriction of $X$ to a neighbourhood of $V \cap S_{\varepsilon}^{2 n-1}$ has no zeros. In [2] it is explained how one can construct a vector field $\widehat{X}$ on a neighbourhood of the boundary $\partial M_{\underline{\delta}}$ of the Milnor fibre approximating $X$ on a neighbourhood of $V \cap S_{\varepsilon}^{2 n-1}$. Two approximations of this sort are close to each other and therefore homotopic to each other in the class of vector fields non-vanishing on a neighbourhood of the boundary $\partial M_{\underline{\delta}}$. Let $\widetilde{X}$ be an extension of $\widehat{X}$ to the Milnor fibre $M_{\underline{\delta}}$ with isolated singular points.

The GSV-index of the vector field $X$ is

$$
\operatorname{ind}_{\mathrm{GSV}}(X ; V, 0)=\sum_{p \in \operatorname{Sing}} \operatorname{ind}\left(\widetilde{X} ; M_{\underline{\delta}}, p\right) .
$$

\section{Burnside rings and Euler characteristics}

Let $G$ be a finite group. Let Conjsub $G$ be the set of conjugacy classes of subgroups of $G$. A $G$-set is a set with an action of the group $G$. A $G$-set is irreducible if the action of $G$ on it is transitive. Isomorphism classes of irreducible $G$-sets are in one-to-one correspondence with the elements of Conjsub $G$ : to the conjugacy class $[H]$ containing a subgroup $H \subset G$ one associates the isomorphism class $[G / H]$ of the $G$-set $G / H$. The Burnside ring $B(G)$ of $G$ is the Grothendieck ring of finite $G$-sets, i.e. the abelian group generated by the isomorphism classes of finite $G$-sets modulo the relation $[A \amalg B]=[A]+[B]$ for finite $G$-sets $A$ and $B$. The multiplication in $B(G)$ is defined 
by the cartesian product. As an abelian group, $B(G)$ is freely generated by the isomorphism classes of irreducible $G$-sets, i.e. each element of $B(G)$ can be written in a unique way as $\sum_{[H] \in \text { Conjsub } G} a_{[H]}[G / H]$ with $a_{[H]} \in \mathbb{Z}$. The element 1 in the ring $B(G)$ is represented by the $G$-set $[G / G]$ consisting of one point (with the trivial $G$-action).

There is a natural homomorphism from the Burnside ring $B(G)$ to the $\operatorname{ring} R(G)$ of representations of the group $G$ which sends a $G$-set $X$ to the (vector) space of functions on $X$. If $G$ is cyclic, then this homomorphism is injective. In general, it is neither injective nor surjective.

For a subgroup $H \subset G$ there are natural maps $\mathrm{R}_{H}^{G}: B(G) \rightarrow B(H)$ and $\mathrm{I}_{H}^{G}: B(H) \rightarrow B(G)$. The restriction map $\mathrm{R}_{H}^{G}$ sends a $G$-set $\mathrm{X}$ to the same set considered with the $H$-action. The induction map $\mathrm{I}_{H}^{G}$ sends an $H$-set $X$ to the product $G \times X$ factorized by the natural equivalence: $\left(g_{1}, x_{1}\right) \equiv\left(g_{2}, x_{2}\right)$ if there exists $g \in H$ such that $g_{2}=g_{1} g, x_{2}=g^{-1} x_{1}$ with the natural (left) $G$-action. Both maps are group homomorphisms, however the induction map $\mathrm{I}_{H}^{G}$ is not a ring homomorphism.

For an action of a group $G$ on a set $X$ and for a point $x \in X$, let $G_{x}=\{g \in G: g x=$ $x\}$ be the isotropy group of the point $x$. For a subgroup $H \subset G$ let $X^{H}=\{x \in X$ : $H x=x\}$ be the fixed point set of the subgroup $H$ and let $X^{(H)}=\left\{x \in X: G_{x}=H\right\}$ be the set of points with the isotropy group $H$. For a conjugacy class $[H] \in \operatorname{Conjsub} G$, let $X^{[H]}=\bigcup_{K \in[H]} X^{K}, X^{([H])}=\bigcup_{K \in[H]} X^{(K)}$.

For a "sufficiently good" topological space $V$, say, a subanalytic variety, its Euler characteristic is

$$
\chi(V)=\sum_{i}(-1)^{i} \operatorname{dim} H_{\mathrm{c}}^{i}(V ; \mathbb{R})
$$

where $H_{\mathrm{c}}^{i}(V ; \mathbb{R})$ are the cohomology groups with compact support. This Euler characteristic is additive (in contrast to the one defined via the usual cohomology groups). For quasi-projective complex analytic varieties both these Euler characteristics coincide.

Let $G$ be a finite group acting on $V$.

Definition 3.1 The equivariant Euler characteristic of $(V, G)$ is the element of the Burnside ring of $G$ defined by

$$
\chi^{G}(V)=\sum_{[H] \in \text { Conjsub } G} \chi\left(V^{([H])} / G\right)[G / H],
$$

see, e.g., $[4,25]$. The reduced equivariant Euler characteristic of $(V, G)$ is

$$
\bar{\chi}^{G}(V)=\chi^{G}(V)-[G / G]
$$

Remark 3.2 For a subgroup $H$ from a conjugacy class [ $H]$, the $G$-action on $V$ induces an $N_{G}(H)$-action on $V^{H}\left(N_{G}(H)\right.$ is the normalizer $\left\{g \in G: g^{-1} H g=H\right\}$ of $\left.H\right)$ and $V^{([H])} / G=V^{(H)} / N_{G}(H)$. Therefore

$$
\chi^{G}(V)=\sum_{[H] \in \text { Conjsub } G} \chi\left(V^{(H)} / N_{G}(H)\right)[G / H] .
$$


Remark 3.3 One can see that the natural homomorphism from $B(G)$ to the $\operatorname{ring} R(G)$ of representations of $G$ described above sends the equivariant Euler characteristic $\chi^{G}(V)$ to the equivariant Euler characteristic $\chi_{G}(V) \in R(G)$ considered, e.g., in [26].

There is a natural homomorphism $|\cdot|$ from the Burnside ring $B(G)$ to $\mathbb{Z}$ sending a (virtual) $G$-set $A$ to the number of elements $|A|$ of $A$. This homomorphism sends the equivariant Euler characteristic $\chi^{G}(V)$ to the usual Euler characteristic $\chi(V)$.

The orbifold Euler characteristic of the pair $(V, G)$ is defined by

$$
\chi^{\text {orb }}(V, G)=\frac{1}{|G|} \sum_{\substack{(g, h) \\ g h=h g}} \chi\left(V^{\langle g, h\rangle}\right)
$$

where $\langle g, h\rangle$ is the subgroup of $G$ generated by $g$ and $h$, see $[1,5,6,20]$. There are higher order Euler characteristics of the pair $(V, G)$ defined by

$$
\chi^{(k)}(V, G)=\frac{1}{|G|} \sum_{\substack{\mathbf{g} \in G^{k+1} \\ g_{i} g_{j}=g_{j} g_{i}}} \chi\left(V^{\left\langle g_{0}, g_{1}, \ldots, g_{k}\right\rangle}\right), \quad k \geq 0,
$$

where $\mathbf{g}=\left(g_{0}, g_{1}, \ldots, g_{k}\right),\left\langle g_{0}, g_{1}, \ldots, g_{k}\right\rangle$ is the subgroup generated by $g_{0}, g_{1}, \ldots$, $g_{k}$, see $[1,3]$. The zeroth order Euler characteristic $\chi^{(0)}(V, G)$ of the pair $(V, G)$ is nothing else but the Euler characteristic of the quotient $V / G$, the first order Euler characteristic $\chi^{(1)}(V, G)$ of the pair $(V, G)$ is just the orbifold one. All these Euler characteristics are additive functions on the Grothendieck ring of subanalytic $G$-sets.

There are homomorphisms $r_{G}^{(k)}$ from $B(G)$ as an abelian group to $\mathbb{Z}$ which send the equivariant Euler characteristic $\chi^{G}(V)$ to the higher order Euler characteristics $\chi^{(k)}(V, G), k \geq 0$. The homomorphism $r_{G}^{(k)}$ is defined by $r_{G}^{(k)}([G / H])=$ $\chi^{(k)}([G / H], G)$. The homomorphism $r_{G}^{(0)}$ sends the class [A] of a finite $G$-set to the number of $G$-orbits in $A$. For an abelian group $G$ one has $\chi^{(k)}([G / H], G)=|H|^{k}$. Therefore one can say that the higher order Euler characteristics are reductions of the equivariant Euler characteristic which is a more general one. The homomorphism $r_{G}^{(k)}$ is not, in general, a ring homomorphism.

Below we construct $G$-equivariant versions of some integer valued invariants as elements of the Burnside ring $B(G)$. They possess the property that the "number of elements" homomorphism $|\cdot|$ sends them to the original invariants (i.e. for trivial $G$ ). Applying the homomorphism $r_{G}^{(k)}$ one gets an orbifold version of each invariant (for $k=1$ ) and also higher order integral invariants. (This was used in [18] to define orbifold versions of the Lefschetz number.)

\section{Equivariant radial index}

We introduce the notion of an equivariant radial index for vector fields. The necessary changes for 1 -forms are clear.

Let $(V, 0)$ be a germ of a closed real subanalytic set with an action of a finite group $G$. (We assume $(V, 0)$ to be embedded into $\left(\mathbb{R}^{N}, 0\right)$ and the $G$-action to be induced by an analytic action on a neighbourhood of $(V, 0)$ in $\left(\mathbb{R}^{N}, 0\right)$. This essentially means 
that the action on $\left(\mathbb{R}^{N}, 0\right)$ can be assumed to be linear, i.e. induced by a representation of $G$.) Let $V=\bigcup_{i=1}^{q} V_{i}$ be a subanalytic Whitney $G$-stratification of $V$. This means that each stratum $V_{i}$ is $G$-invariant, the isotropy subgroups $G_{p}=\{g \in G: g p=p\}$ of all points $p$ of $V_{i}$ are conjugate to each other, and the quotient of the stratum $V_{i}$ by the group $G$ is connected. (Such a stratification can be obtained from an arbitrary subanalytic Whitney stratification of $(V, 0)$ by taking intersections/unions of the strata and of their transforms by the elements of $G$.)

Let $X$ be a $G$-invariant stratified vector field on $(V, 0)$ with an isolated singular point (zero) at the origin. One can show that there exists a (continuous) $G$-invariant stratified vector field $\widetilde{X}$ on $V$ satisfying conditions (a)-(c) from Sect. 2.

Let $A$ be the set (a $G$-set) of the singular points of the vector field $\widetilde{X}$ on $V \cap B_{\varepsilon}$ considered with the multiplicities equal to the usual indices ind $\left(\widetilde{X}_{\mid V_{(p)}} ; V_{(p)}, p\right)$ of the restrictions of the vector field $\widetilde{X}$ to the corresponding strata (smooth manifolds).

Definition 4.1 The equivariant radial index $\operatorname{ind}_{\operatorname{rad}}^{G}(X ; V, 0)$ of the vector field $X$ on $V$ at the origin is the class $[A] \in B(G)$ of the set $A$ of singular points of $\widetilde{X}$ with multiplicities.

Remark 4.2 One can rewrite the definition as

$$
\operatorname{ind}_{\mathrm{rad}}^{G}(X ; V, 0)=\sum_{\bar{p} \in(\operatorname{Sing} \widetilde{X}) / G} \operatorname{ind}\left(\widetilde{X}_{\mid V_{(p)}} ; V_{(p)}, p\right)[G p],
$$

where $p$ is a point of the preimage of $\bar{p}$ under the canonical projection, $[G p]$ is the class of the orbit $G p$ of $p$ in the Burnside ring.

For a subgroup $H \subset G$, the vector field $X$ is $H$-invariant and one has $\operatorname{ind}_{\mathrm{rad}}^{H}(X ; V, 0)=\mathrm{R}_{H}^{G}\left(\operatorname{ind}_{\mathrm{rad}}^{G}(X ; V, 0)\right)$.

The homomorphisms $r_{G}^{(k)}$ give "higher order Euler characteristic versions" of the equivariant index: $\operatorname{ind}_{\mathrm{rad}}^{G,(k)}(X ; V, 0)=r_{G}^{(k)}\left(\operatorname{ind}_{\mathrm{rad}}^{G}(X ; V, 0)\right)$. For $k=1$ one gets an orbifold index of $X$.

For a stratum $V_{i}$ of the stratification, let $\left[G_{i}\right] \in \operatorname{Conjsub} G$ be the conjugacy class of the isotropy subgroups of points of $V_{i}$, and let $G_{i}$ be a representative of it. Singular points of the vector field $\widetilde{X}$ on the stratum $V_{i}$ are distributed between the orbits of the $G$-action, all of which (as $G$-sets) are isomorphic to $\left[G / G_{i}\right]$. Therefore

$$
\operatorname{ind}_{\mathrm{rad}}^{G}(X ; V, 0)=\sum_{i=1}^{q} \frac{\left|G_{i}\right|}{|G|} \operatorname{ind}\left(X ; V_{i}, 0\right)\left[G / G_{i}\right],
$$

see Proposition 2.1.

Let us write the equivariant index $\operatorname{ind}_{\mathrm{rad}}^{G}(X ; V, 0)$ in terms of the usual (nonequivariant) indices of the vector field $X$ on the fixed point sets $V^{H}$ of subgroups of $G$ or on the "fixed point sets" $V^{[H]}=\bigcup_{K \in[H]} V^{K}$ of conjugacy classes of subgroups of $G$.

Assume that

$$
\operatorname{ind}_{\operatorname{rad}}^{G}(X ; V, 0)=\sum_{[H] \in \text { Conjsub } G} a_{[H]}[G / H] .
$$


One has

$$
\operatorname{ind}_{\operatorname{rad}}(X ; V, 0)=\sum_{[H] \in \text { Conjsub } G} a_{[H]} \frac{|G|}{|H|} .
$$

Equation (1) implies that

$$
\operatorname{ind}_{\mathrm{rad}}^{G}\left(X ; V^{[H]}, 0\right)=\sum_{[K] \geq[H]} a_{[K]}[G / K]
$$

and therefore

$$
\operatorname{ind}_{\mathrm{rad}}\left(X ; V^{[H]}, 0\right)=\sum_{[K] \geq[H]} a_{[K]} \frac{|G|}{|K|} .
$$

Equation (2) can be rewritten as

$$
\operatorname{ind}_{\operatorname{rad}}\left(X ; V^{[H]}, 0\right)=\sum_{[K] \in \text { Conjsub } G} \zeta([H],[K]) a_{[K]} \frac{|G|}{|K|},
$$

where

$$
\zeta([H],[K])= \begin{cases}0 & \text { for }[K] \geq[H], \\ 1 & \text { for }[K] \geq[H]\end{cases}
$$

is the zeta function of the partially ordered set Conjsub $G$. (One has $[K] \geq[H]$ if there exist representatives $K$ and $H$ of $[K]$ and $[H]$ respectively such that $K \supset H$.) The system (3) is a triangular system of linear equations with respect to $a_{[H]}$. Its solution gives the following statement.

Proposition 4.3 One has

$$
\begin{aligned}
& \operatorname{ind}_{\mathrm{rad}}^{G}(X ; V, 0) \\
&=\sum_{[H] \in \text { Conjsub } G} \frac{|H|}{|G|}\left(\sum_{[K] \in \operatorname{Conjsub} G} \mu([H],[K]) \operatorname{ind}_{\operatorname{rad}}\left(X ; V^{[K]}, 0\right)\right)[G / H],
\end{aligned}
$$

where $\mu([H],[K])$ is the Möbius function of the poset Conjsub $G$, see, e.g, [19].

In a similar way one has

$$
\operatorname{ind}_{\mathrm{rad}}\left(X ; V^{H}, 0\right)=\sum_{K \geq H} a_{[K]} \frac{\left|N_{G}(K)\right|}{|K|},
$$

where $N_{G}(K)$ is the normalizer $\left\{g \in G: g^{-1} K g=K\right\}$ of the subgroup $K$. This can be rewritten as

$$
\operatorname{ind}_{\mathrm{rad}}\left(X ; V^{H}, 0\right)=\sum_{K \in \operatorname{Sub} G} \zeta^{\prime}(H, K) a_{[K]} \frac{\left|N_{G}(K)\right|}{|K|}
$$


where $\zeta^{\prime}(H, K)$ is the zeta function of the partially ordered set Sub $G$ of subgroups of $G$. Thus one gets the following statement.

Proposition 4.4 One has

$$
\begin{aligned}
\operatorname{ind}_{\mathrm{rad}}^{G}(X ; V, 0) & \\
= & \sum_{[H] \in \text { Conjsub } G} \frac{|H|}{\left|N_{G}(H)\right|}\left(\sum_{K \in \operatorname{Sub} G} \mu^{\prime}(H, K) \operatorname{ind}_{\mathrm{rad}}\left(X ; V^{K}, 0\right)\right)[G / H],
\end{aligned}
$$

where $\mu^{\prime}(H, K)$ is the Möbius function of the poset Sub G.

Let $V$ be a locally compact subanalytic $G$-variety and let $X$ be a $G$-invariant (stratified with respect to a stratification) vector field on $V$ with isolated singular points. Let $p$ be a singular point of $X$. The germ $(V, p)$ carries an action of the isotropy subgroup $G_{p}$ of $p$. Therefore the index $\operatorname{ind}_{\text {rad }}^{G_{p}}(X ; V, p) \in B\left(G_{p}\right)$ is defined.

Definition 4.5 The G-equivariant index $\operatorname{ind}_{\mathrm{rad}}^{G}(X ; V, G p)$ of the vector field $X$ at the orbit $G p$ is $\mathrm{I}_{G}^{G}\left(\operatorname{ind}_{\mathrm{rad}}^{G_{p}}(X ; V, p)\right) \in B(G)$.

One can see that the $G$-equivariant index of a vector field (and of a 1-form as well) satisfies the law of conservation of number: for a $(G$-invariant) deformation $\widetilde{X}$ of a vector field $X$ the sum of the $G$-equivariant indices of $\widetilde{X}$ at the orbits in a neighbourhood of an orbit $G p$ is equal to $\operatorname{ind}_{\text {rad }}^{G}(X ; V, G p)$.

One has the following equivariant version of the Poincaré-Hopf theorem.

Theorem 4.6 Let $V=\bigcup_{i=1}^{q} V_{i}$ be a compact subanalytic variety and let $X$ be a $G$-invariant stratified vector field on $V$ with isolated singular points. Then the sum of the indices of the vector field $X$ over all singular orbits is equal to the G-equivariant Euler characteristic of $V$ :

$$
\sum_{\bar{p} \in(\operatorname{Sing} X) / G} \operatorname{ind}_{\mathrm{rad}}^{G}\left(X ; V, \pi^{-1}(\bar{p})\right)=\chi^{G}(V) \in B(G)
$$

( $\pi: V \rightarrow V / G$ is the canonical projection).

An analogous statement holds for 1-forms.

Proof Let $\tilde{X}$ be a $G$-invariant deformation of $X$ which differs from $X$ only in neighbourhoods of singular points and which satisfies the properties of the definition of the equivariant index. One has

$$
\begin{aligned}
\sum_{\bar{p} \in(\operatorname{Sing} X) / G} \operatorname{ind}_{\mathrm{rad}}^{G}\left(X ; V, \pi^{-1}(\bar{p})\right) & =\sum_{\bar{p} \in(\operatorname{Sing} X) / G} \operatorname{ind}_{\mathrm{rad}}^{G}\left(\tilde{X} ; V, \pi^{-1}(\bar{p})\right) \\
& =\sum_{i=1}^{q} \sum_{p \in V_{i}} \frac{\left|G_{i}\right|}{|G|} \operatorname{ind}\left(\widetilde{X} ; V_{i}, p\right)\left[G / G_{i}\right],
\end{aligned}
$$




$$
\chi^{G}(V)=\sum_{i=1}^{q} \frac{\left|G_{i}\right|}{|G|} \chi\left(V_{i}\right)\left[G / G_{i}\right]
$$

As in the proof of Proposition 2.1, let $W_{i}$ be the complement in $V_{i}$ of a small tubular neighbourhood of its boundary $\partial V_{i}$. It can be regarded as a manifold with boundary. On the boundary $\partial W_{i}$ the restriction $\widetilde{X}_{\mid W_{i}}$ of the vector field $\widetilde{X}$ is homotopic, in the class of vector fields non-vanishing on $\partial W_{i}$, to a vector field pointing inwards. Therefore

$$
\sum_{p \in V_{i}} \operatorname{ind}\left(\tilde{X} ; V_{i}, p\right)=\chi\left(W_{i} \backslash \partial W_{i}\right)=\chi\left(V_{i}\right)
$$

(We use the fact that, on a compact manifold with boundary, the sum of the indices of the singular points of a vector field pointing inwards on the boundary is equal to the Euler characteristic of the interior of the manifold.)

Example 4.7 The radial index of the differential of a function on a singular variety (up to sign) can be regarded as an analogue of the Milnor number of the function germ. The equivariant index gives an equivariant analogue of the Milnor number. Let $(V, 0)$ be a germ of a complex analytic $G$-variety of pure dimension $n$ and let $f:(V, 0) \rightarrow(\mathbb{C}, 0)$ be a germ of a $G$-invariant complex analytic function. The germ $f$ is the lifting $\bar{f} \circ \pi$ of an analytic function germ $\bar{f}:(V / G, 0) \rightarrow(\mathbb{C}, 0)(\pi: V \rightarrow V / G$ is the canonical projection). The Milnor fibres $M_{\operatorname{Re} f}^{+}$and $M_{\operatorname{Re} f}^{-}$of $\operatorname{Re} f$ are $G$-homotopy equivalent to the Milnor fibre $f^{-1}(\delta) \cap B_{\varepsilon}$ of $f$ and one has ind ${ }_{\text {rad }}^{G}(d f ; V, 0)=-\bar{\chi}^{G}\left(M_{f}\right)$. The

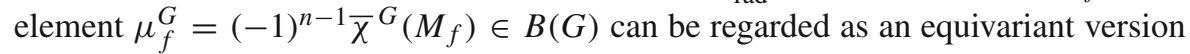
of the Milnor number of $f$. The homomorphism $r_{G}^{(0)}$ from $B(G)$ to $\mathbb{Z}$ which sends $[G / H]$ to 1 maps the element $\mu_{f}^{G}$ to the Milnor number of the germ $\bar{f}$ on $(V / G, 0)$ defined (for smooth $V / G$ ) in [26].

Let $(V, 0)$ be a germ of a closed subanalytic $G$-variety, let $(V / G, 0)$ be the quotient by the $G$-action, let $\pi: V \rightarrow V / G$ be the canonical projection, and let $V=\bigcup_{i=1}^{q} V_{i}$ and $V / G=\bigcup_{i=1}^{q} V_{i} / G$ be Whitney stratifications of $V$ and $V / G$ respectively compatible with the partitions corresponding to different orbit types. For a stratum $V_{i}$, $i=1, \ldots, q$, let $\left[G_{i}\right]$ be the conjugacy class of the isotropy subgroups of points of $V_{i}$ and let $G_{i}$ be a representative of it. Let $X$ be a $G$-invariant stratified vector field on $V$ with an isolated singular point at 0 . The image of $X$ under the map $\pi_{*}$ defines a stratified vector field $\bar{X}$ on $V / G$ with an isolated singular point. One can easily see that a product/cone-like structure in a neighbourhood of a point $\bar{p} \in V_{i} / G$ defines a $G_{p}$-invariant product/cone-like structure in a neighbourhood of a point $p \in \pi^{-1}(\bar{p})$. This implies that, if $\widetilde{X}$ is an appropriate (in the sense of the definition of the radial index) deformation of the vector field $X$, then $\pi_{*} \widetilde{X}$ is an appropriate deformation of the vector field $\bar{X}$. Therefore one has the following statement.

\section{Proposition 4.8}

$$
\operatorname{ind}_{\mathrm{rad}}^{G}(X ; V, 0)=\sum_{[H] \in \text { Conjsub } G} \operatorname{ind}_{\mathrm{rad}}\left(\bar{X} ; V^{([H])} / G, 0\right)[G / H],
$$


where $\operatorname{ind}_{\mathrm{rad}}\left(\bar{X} ; V^{([H])} / G, 0\right)$ is the (radial) index of the vector field $\bar{X}$ on the nonclosed variety $V^{([H])} / G$ defined in Sect. 2.

Let $\omega$ be a 1 -form on $V / G$ with an isolated singular point at 0 . The 1 -form $\pi^{*} \omega$ on $V$ is $G$-invariant and has an isolated singular point at 0 . One can easily see that the following analogue of Proposition 4.8 holds.

Proposition $4.9 \operatorname{ind}_{\mathrm{rad}}^{G}\left(\pi^{*} \omega ; V, 0\right)=\sum_{[H] \in \operatorname{Conjsub} G} \operatorname{ind}_{\operatorname{rad}}\left(\omega ; V^{([H])} / G, 0\right)[G / H]$.

Example 4.10 Assume that $f:(V, 0) \rightarrow(\mathbb{R}, 0)$ is a $G$-invariant germ of an analytic function on $(V, 0)$ with an isolated critical point at 0 . (This means that the differential $d f$ of $f$ has an isolated singular point at 0 .) For a small enough positive $\varepsilon$ the restriction of $f$ to $V \cap B_{\varepsilon}$ is a locally trivial fibration over a punctured neighbourhood of zero in $\mathbb{R}$, in general with different fibres over the positive and the negative part. The fibres ( $G$-varieties) $M_{f}^{+}$and $M_{f}^{-}$of this fibration can be called positive and negative Milnor fibres of $f$ respectively. One has the following generalization of [14, Theorem 2].

Proposition 4.11 $\operatorname{ind}_{\mathrm{rad}}^{G}(d f ; V, 0)=-\bar{\chi}^{G}\left(M_{f}^{-}\right)$.

For a $(G$-invariant) complex analytic function $f:(V, 0) \rightarrow(\mathbb{C}, 0)$ on a germ of a complex analytic variety, the Milnor fibres $M_{f}^{ \pm}$of its real part are homeomorphic to the product $M_{f} \times I$, where $M_{f}=f^{-1}(\delta) \cap B_{\varepsilon}$ is the Milnor fibre of $f(0<|\delta| \ll \varepsilon$ small enough), $I$ is the segment [0,1]; see [17, Proposition 2.A.3]. Therefore in this case

$$
\operatorname{ind}_{\mathrm{rad}}^{G}(d f ; V, 0)=-\bar{\chi}^{G}\left(M_{f}\right) .
$$

Example 4.12 Let the complex vector space $\mathbb{C}^{n}$ be equipped with a (linear) $G$-action and let $\omega=\sum_{i=1}^{n} A_{i}(z) d z_{i}$ be a complex analytic $G$-invariant 1 -form on $\left(\mathbb{C}^{n}, 0\right)$ with an isolated singular point at the origin. For a subgroup $K \subset G$, let $n_{K}$ be the dimension of the fixed point set $\left(\mathbb{C}^{n}\right)^{K}$ of $K$ (a vector subspace). The (radial) index $\operatorname{ind}_{\mathrm{rad}}\left(\omega ;\left(\mathbb{C}^{n}\right)^{K}, 0\right)$ is $(-1)^{n_{K}}$ times the dimension of the space $\Omega_{\left(\mathbb{C}^{n}\right)^{K}, \omega}=$ $\Omega_{\left(\mathbb{C}^{n}\right)^{K}, 0}^{n_{K}} /\left(\Omega_{\left(\mathbb{C}^{n}\right)^{K}, 0}^{n_{K}-1} \wedge \omega_{\mid\left(\mathbb{C}^{n}\right)^{K}}\right)$, where $\Omega_{\left(\mathbb{C}^{n}\right)^{K}, 0}^{m}$ is the vector space of germs of analytic differential $m$-forms on $\left(\left(\mathbb{C}^{n}\right)^{K}, 0\right)$. Together with Proposition 4.4 this gives

$$
\begin{aligned}
& \operatorname{ind}_{\mathrm{rad}}^{G}\left(\omega ; \mathbb{C}^{n}, 0\right) \\
& \quad=\sum_{[H] \in \text { Conjsub } G} \frac{|H|}{\left|N_{G}(H)\right|}\left(\sum_{K \in \operatorname{Sub} G}(-1)^{n_{K}} \mu^{\prime}(H, K) \operatorname{dim} \Omega_{\left(\mathbb{C}^{n}\right)^{K}, \omega}\right)[G / H] .
\end{aligned}
$$

Example 4.13 Let $f\left(z_{1}, \ldots, z_{n}\right)=\sum_{i=1}^{n} \prod_{j=1}^{n} z_{i}^{E_{i j}}$ be an invertible polynomial (i.e. $f$ is a quasi-homogeneous polynomial, $E_{i j} \in \mathbb{Z}, E_{i j} \geq 0, \operatorname{det} E_{i j} \neq 0$ ) with an isolated critical point at the origin. Let

$$
G_{f}=\left\{\left(\lambda_{1}, \ldots, \lambda_{n}\right) \in \mathbb{C}^{n}: f\left(\lambda_{1} z_{1}, \ldots, \lambda_{n} z_{n}\right)=f\left(z_{1}, \ldots, z_{n}\right)\right\}
$$


be the group of diagonal symmetries of $f$. The Berglund-Hübsch dual polynomial to $f$ is $\widetilde{f}\left(z_{1}, \ldots, z_{n}\right)=\sum_{i=1}^{n} \prod_{j=1}^{n} z_{i}^{E_{j i}}$. The group $G_{\tilde{f}}$ of diagonal symmetries of $\tilde{f}$ is (canonically) isomorphic to $G_{f}^{*}=\operatorname{Hom}\left(G, \mathbb{C}^{*}\right)$ [12]. The result of [12] together with Proposition 4.11 says that the indices ind ${ }_{\text {rad }}^{G_{f}}\left(d f ; \mathbb{C}^{n}, 0\right)$ and $\operatorname{ind}_{\mathrm{rad}}^{G}\left(d \tilde{f} ; \mathbb{C}^{n}, 0\right)$ are Saito dual to each other in the sense of [12]. Let $\bar{f}$ and $\overline{\widetilde{f}}$ be the functions on $\mathbb{C}^{n} / G_{f}$ and $\mathbb{C}^{n} / G_{\tilde{f}}$ corresponding to $f$ and $\widetilde{f}$ respectively. The Saito dual elements of the Burnside rings $B\left(G_{f}\right)$ and $B\left(G_{\tilde{f}}\right)$ have the same images under $r_{G_{f}}^{(0)}$ and $r_{G_{\tilde{f}}}^{(0)}$ respectively. Therefore the indices $\operatorname{ind}_{\mathrm{rad}}^{G_{f},(0)}\left(d f ; \mathbb{C}^{n}, 0\right)=\operatorname{ind}_{\mathrm{rad}}\left(d \bar{f} ; \mathbb{C}^{n} / G_{f}, 0\right)$ and $\operatorname{ind}_{\operatorname{rad}}^{G},\left(d \tilde{f} ; \mathbb{C}^{n}, 0\right)=\operatorname{ind}_{\operatorname{rad}}\left(d \overrightarrow{\widetilde{f}} ; \mathbb{C}^{n} / G_{\tilde{f}}, 0\right)$ (equal to the minus reduced Euler characteristics of $M_{f} / G_{f}$ and $M_{\tilde{f}} / G_{\tilde{f}}$ respectively) coincide. For a subgroup $H \subset G_{f}$ its dual $H^{T} \subset G_{\tilde{f}}$ is the kernel of the natural map $i^{*}: G_{f}^{*} \rightarrow H^{*}$ induced by the inclusion $i: H \rightarrow G_{f}$. The result of [13] says that the orbifold indices $\operatorname{ind}_{\mathrm{rad}}^{H,(1)}\left(d f ; \mathbb{C}^{n}, 0\right)$ and ind $\mathrm{rad}^{T},(1)\left(d \widetilde{f} ; \mathbb{C}^{n}, 0\right)$ coincide.

\section{Equivariant GSV-index}

Let $\left(\mathbb{C}^{n}, 0\right)$ be endowed by a $G$-action. (Without loss of generality one may assume that the action is linear, i.e. it is defined by a representation $G \rightarrow \operatorname{GL}(n)$.) Let $f_{1}, \ldots, f_{k}$ be $G$-invariant holomorphic function germs on $\left(\mathbb{C}^{n}, 0\right)$ defining a complete intersection singularity $(V, 0)=\left\{f_{1}=\cdots=f_{k}=0\right\}$. We assume that a neighbourhood of the origin in $\mathbb{C}^{n}$ permits a Whitney stratification adapted to $V$ and satisfying the Thom $a_{f}$ condition.

Let $X$ be a $G$-invariant stratified vector field on $(V, 0)$ with an isolated singular point at the origin. In particular, the restriction of $X$ to a neighbourhood of $V \cap S_{\mathcal{E}}^{2 n-1}$ has no zeros. The vector fields $\widehat{X}$ and $\widetilde{X}$ described in Sect. 2 can be made $G$-invariant. (To get a $G$-invariant vector field, one can take an arbitrary one and take the mean over the group.)

Definition 5.1 The G-equivariant $G S V$-index of the vector field $X$ is

$$
\operatorname{ind}_{\mathrm{GSV}}^{G}(X ; V, 0)=\sum_{\bar{p} \in(\operatorname{Sing} \widetilde{X}) / G} \operatorname{ind}_{\mathrm{rad}}^{G}\left(\widetilde{X} ; M_{\underline{\delta}}, \pi^{-1}(\bar{p})\right) \in B(G),
$$

where $\pi: M_{\underline{\delta}} \rightarrow M_{\underline{\delta}} / G$ is the canonical projection.

It is easy to show that the right hand side of (4) does not depend on the extension $\widetilde{X}$ of $\widehat{X}$ and therefore the equivariant GSV-index is well-defined.

A compact complex analytic $G$-variety $V$, all singular points of which are ICIS, has a natural smoothing $\widetilde{V}$. (The manifold $V$ is in general $C^{\infty}$, not necessarily complex analytic. It can be chosen to be complex analytic if $V$ is a projective complete intersection with isolated singularities.) Let $X$ be a $G$-invariant vector field on $V$ with isolated singular points. For $p \in \operatorname{Sing} X$, the $G$-equivariant GSV-index of $X$ at the orbit $G p$ is defined as 


$$
\operatorname{ind}_{\mathrm{GSV}}^{G}(X ; V, G p)=\mathrm{I}_{G_{p}}^{G}\left(\operatorname{ind}_{\mathrm{GSV}}^{G_{p}}(X ; V, p)\right) .
$$

Since the GSV-index counts singular points of the vector field on the smoothing of the ICIS, one has the following version of the Poincaré-Hopf theorem.

Proposition 5.2 The sum of the equivariant GSV-indices of the singular orbits of $X$ on $V$ is equal to the equivariant Euler characteristic of $\widetilde{V}$ :

$$
\sum_{G p \in(\operatorname{Sing} X) / G} \operatorname{ind}_{\mathrm{GSV}}^{G}(X ; V, G p)=\chi^{G}(\widetilde{V}) \in B(G) .
$$

A relation between the equivariant GSV-index and the radial one on an ICIS can be described in the following way. The Milnor fibre $M_{\delta}$ is a manifold with a $G$-action and therefore its equivariant Euler characteristic $\chi^{G}\left(M_{\underline{\delta}}\right) \in B(G)$ is defined. Let $\bar{\chi}^{G}\left(M_{\underline{\delta}}\right)=\chi^{G}\left(M_{\underline{\delta}}\right)-1$ be the reduced equivariant Euler characteristic of $M_{\underline{\delta}}$. (The element $(-1)^{n-k} \bar{\chi}^{G}\left(M_{\underline{\delta}}\right) \in B(G)$ can be regarded as an equivariant version of the Milnor number of the ICIS $(V, 0)$.)

Proposition $5.3 \operatorname{ind}_{\mathrm{GSV}}^{G}(X ; V, 0)=\operatorname{ind}_{\mathrm{rad}}^{G}(X ; V, 0)+\bar{\chi}^{G}\left(M_{\underline{\delta}}\right)$.

Proof This follows from the following facts: 1) both the GSV-index and the radial one satisfy the law of conservation of number, and 2) for the radial vector field $X_{\text {rad }}$ on $(V, 0)$ one has ind ${ }^{G}\left(X_{\mathrm{rad}} ; V, 0\right)=1, \operatorname{ind}_{\mathrm{GSV}}^{G}\left(X_{\mathrm{rad}} ; V, 0\right)=\chi^{G}\left(M_{\underline{\delta}}\right)$.

Analogous notions and statements exist for $G$-invariant 1 -forms on complete intersection singularities.

Example 5.4 Let $(V, 0)=\left\{f_{1}=\cdots=f_{k}=0\right\}$ be an ICIS. The equivariant Euler characteristic $\chi^{G}\left(M_{\underline{\delta}}\right)$ of the Milnor fibre of $(V, 0)$ is equal to the sum of the equivariant indices of the $G$-orbits of singular points on $M_{\underline{\delta}}$ of a radial 1-form on $V$. Applying Proposition 4.4 to each of the indices one gets:

$$
\chi^{G}\left(M_{\underline{\delta}}\right)=\sum_{[H] \in \text { Conjsub } G} \frac{|H|}{\left|N_{G}(H)\right|}\left(\sum_{K \in \operatorname{Sub} G} \mu^{\prime}(H, K) \chi\left(M_{\underline{\delta}}\right)\right)[G / H] .
$$

Since $\sum_{K} \mu^{\prime}(H, K)=\sum_{K} \zeta(K, G) \mu^{\prime}(H, K)=\delta(H, G)$, where $\delta(\cdot, \cdot)$ is the Kronecker delta function, one has

$$
\bar{\chi}^{G}\left(M_{\underline{\delta}}\right)=\sum_{[H] \in \text { Conjsub } G} \frac{|H|}{\left|N_{G}(H)\right|}\left(\sum_{K \in \operatorname{Sub} G} \mu^{\prime}(H, K) \bar{\chi}\left(M_{\underline{\delta}}\right)\right)[G / H] .
$$

Let $\omega$ be a holomorphic $G$-invariant 1 -form with an isolated singular point at the origin. If the dimension of the fixed point set $\left(\mathbb{C}^{n}\right)^{K}$ of a subgroup $K \subset G$ is greater than $k$, then the GSV-index $\operatorname{ind}_{\mathrm{GSV}}\left(\omega ;\left(\mathbb{C}^{n}\right)^{K}, 0\right)$ is $(-1)^{n_{K}-k}$ times the dimension of the space $\Omega_{V^{K}, \omega}=\Omega_{V^{K}, 0}^{n_{K}-k} /\left(\omega \wedge \Omega_{V^{K}, 0}^{n_{K}-k-1}\right)$, where $\Omega_{V^{K}, 0}^{s}=$ $\Omega_{\left(\mathbb{C}^{n}\right)^{K}, 0}^{s} /\left(f_{i} \Omega_{\left(\mathbb{C}^{n}\right)^{K}, 0}^{s}, d f_{i} \wedge \Omega_{\left(\mathbb{C}^{n}\right)^{K}, 0}^{s-1}\right)[8,10]$. 
Propositions 4.4, 5.3 and (5) imply that

$$
\begin{aligned}
& \operatorname{ind}_{\mathrm{GSV}}^{G}(\omega ; V, 0) \\
& =\sum_{[H] \in \operatorname{Conjsub} G} \frac{|H|}{\left|N_{G}(H)\right|}\left(\sum_{\substack{K \in \operatorname{Sub} G \\
\operatorname{dim}\left(\mathbb{C}^{n}\right)^{K}>k}} \mu^{\prime}(H, K) \operatorname{dim} \Omega_{V^{K}, \omega}\right)[G / H] .
\end{aligned}
$$

\section{References}

1. Atiyah, M., Segal, G.: On equivariant Euler characteristics. J. Geom. Phys. 6(4), 671-677 (1989)

2. Brasselet, J.-P., Seade, J., Suwa, T.: Vector Fields on Singular Varieties. Lecture Notes in Mathematics, vol. 1987. Springer, Berlin (2009)

3. Bryan, J., Fulman, J.: Orbifold Euler characteristics and the number of commuting $m$-tuples in the symmetric groups. Ann. Comb. 2(1), 1-6 (1998)

4. tom Dieck, T.: Transformation Groups and Representation Theory. Lecture Notes in Mathematics, vol. 766. Springer, Berlin (1979)

5. Dixon, L., Harvey, J.A., Vafa, C., Witten, E.: Strings on orbifolds. Nuclear Phys. B 261(4), 678-686 (1985)

6. Dixon, L., Harvey, J.A., Vafa, C., Witten, E.: Strings on orbifolds (II). Nuclear Phys. B 274(2), 285-314 (1986)

7. Dutertre, N.: Radial index and Poincaré-Hopf index of 1-forms on semi-analytic sets. Math. Proc. Camb. Philos. Soc. 148(2), 297-330 (2010)

8. Ebeling, W., Gusein-Zade, S.M.: Indices of 1-forms on an isolated complete intersection singularity. Mosc. Math. J. 3(2), 439-455 (2003)

9. Ebeling, W., Gusein-Zade, S.M.: Radial index and Euler obstruction of a 1-form on a singular variety. Geom. Dedicata 113, 231-241 (2005)

10. Ebeling, W., Gusein-Zade, S.M.: Quadratic forms for a 1-form on an isolated complete intersection singularity. Math. Z. 252(4), 755-766 (2006)

11. Ebeling, W., Gusein-Zade, S.M.: Indices of vector fields and 1-forms on singular varieties. In: Catanese, F., Esnault, H., Huckleberry, A., Hulek, K., Peternell, T. (eds.) Global Aspects of Complex Geometry, pp. 129-169. Springer, Berlin (2006)

12. Ebeling, W., Gusein-Zade, S.M.: Saito duality between Burnside rings for invertible polynomials. Bull. Lond. Math. Soc. 44(4), 814-822 (2012)

13. Ebeling, W., Gusein-Zade, S.M.: Orbifold Euler characteristics for dual invertible polynomials. Mosc. Math. J. 12(1), 49-54 (2012)

14. Ebeling, W., Gusein-Zade, S.M., Seade, J.: Homological index for 1-forms and a Milnor number for isolated singularities. Int. J. Math. 15(9), 895-905 (2004)

15. Gómez-Mont, X.: An algebraic formula for the index of a vector field on a hypersurface with an isolated singularity. J. Algebraic Geom. 7(4), 731-752 (1998)

16. Gómez-Mont, X., Seade, J., Verjovsky, A.: The index of a holomorphic flow with an isolated singularity. Math. Ann. 291(4), 737-751 (1991)

17. Goresky, M., MacPherson, R.: Stratified Morse Theory. Ergebnisse der Mathematik und ihrer Grenzgebiete (3), vol. 14. Springer, Berlin (1988)

18. Gusein-Zade, S.M., Luengo, I., Melle-Hernández, A.: On an equivariant version of the zeta function of a transformation. Arnold Math. J. (2012). arXiv:1203.3344

19. Hall Jr, M.: Combinatorial Theory. Wiley-Interscience Series in Discrete Mathematics, 2nd edn. John Wiley \& Sons, New York (1986)

20. Hirzebruch, F., Höfer, Th: On the Euler number of an orbifold. Math. Ann. 286(1-3), 255-260 (1990)

21. Lück, W., Rosenberg, J.: The equivariant Lefschetz fixed point theorem for proper cocompact $G$ manifolds. In: Farrell, F.T., Lück, W. (eds.) High-Dimensional Manifold Topology, pp. 322-361. World Scientific, River Edge (2003)

22. MacPherson, R.D.: Chern classes for singular algebraic varieties. Ann. Math. 100(2), 423-432 (1974)

23. Schwartz, M.-H.: Classes caractéristiques définies par une stratification d'une variété analytique complexe. C. R. Acad. Sci. Paris Sér. I Math. 260, 3262-3264, 3535-3537 (1965) 
24. Seade, J.A., Suwa, T.: A residue formula for the index of a holomorphic flow. Math. Ann. 304(4), 621-634 (1996)

25. Verdier, J.-L.: Caractéristique d'Euler-Poincaré. Bull. Soc. Math. Fr. 101, 441-445 (1973)

26. Wall, C.T.C.: A note on symmetry of singularities. Bull. Lond. Math. Soc. 12(3), 169-175 (1980) 